\title{
First detection of autochthonous extensively drug-resistant NDM-1 Pseudomonas aeruginosa ST235 from a patient with bloodstream infection in Italy, October 2019
}

Daniela Loconsole ${ }^{1 \dagger}$, Marisa Accogli ${ }^{1 \dagger}$, Monica Monaco ${ }^{2}$, Maria Del Grosso ${ }^{2}$, Anna Lisa De Robertis ${ }^{1}$, Anna Morea', Loredana Capozzi ${ }^{3}$, Laura Del Sambro ${ }^{3}$, Annarosa Simone ${ }^{4}$, Vincenzo De Letteriis ${ }^{4}$, Michele Quarto', Antonio Parisi ${ }^{3}$ and Maria Chironna ${ }^{1 *}$ (D)

\begin{abstract}
Background: Pseudomonas aeruginosa (PA) is one of the most common and serious causes of healthcareassociated bacteremia. The emergence and dissemination of multidrug-resistant (MDR) and extensively drugresistant (XDR) PA strains pose a major clinical concern. ST235-PA is a high-risk clone which shows a high capacity to acquire antibiotic resistance. Here we describe the first autochthonous New Delhi metallo-ß-lactamase (NDM)producing Pseudomonas aeruginosa ST235 identified in Italy.
\end{abstract}

Case presentation: In October 2019, a patient residing in an elderly health care and rehabilitation facility, was hospitalized and died from sepsis caused by an XDR-PA. The strain belonged to the high-risk clone sequence type ST235. Whole genome sequencing (WGS) revealed the presence of genes encoding NDM-1 and multiple $\beta$ lactamases, many clinically significant multidrug efflux pump complexes and also the virulence gene ExoU, which is associated with a high cytotoxic phenotype.

Conclusions: Few strains of NDM-1-PA have been identified worldwide, all belonging to ST235. The combination of ST235 and EXOU is a predictor of highly unfavorable prognosis. The potential spread of these high-risk clones in healthcare settings is worrisome because treatment options are limited. Early identification of high-risk clones could help in outbreaks investigation and infections control.

Keywords: Pseudomonas aeruginosa, Extensively-drug resistant, New Delhi metallo beta-, lactamase, Whole-genome sequencing, ST235, sepsis

\footnotetext{
* Correspondence: maria.chironna@uniba.it

${ }^{\dagger}$ Daniela Loconsole and Marisa Accogli contributed equally to this work.

'Department of Biomedical Sciences and Human Oncology, Hygiene Unit,

University of Bari "Aldo Moro", P.zza G. Cesare 11, 70124 Bari, Italy

Full list of author information is available at the end of the article
}

C The Author(s). 2020 Open Access This article is licensed under a Creative Commons Attribution 4.0 International License, which permits use, sharing, adaptation, distribution and reproduction in any medium or format, as long as you give appropriate credit to the original author(s) and the source, provide a link to the Creative Commons licence, and indicate if changes were made. The images or other third party material in this article are included in the article's Creative Commons licence, unless indicated otherwise in a credit line to the material. If material is not included in the article's Creative Commons licence and your intended use is not permitted by statutory regulation or exceeds the permitted use, you will need to obtain permission directly from the copyright holder. To view a copy of this licence, visit http://creativecommons.org/licenses/by/4.0/. The Creative Commons Public Domain Dedication waiver (http://creativecommons.org/publicdomain/zero/1.0/) applies to the data made available in this article, unless otherwise stated in a credit line to the data. 


\section{Background}

Pseudomonas aeruginosa (PA) is an opportunistic human pathogen implicated in various acute and chronic infections, including infections of the respiratory, urinary, and gastrointestinal tracts, as well as bacteremia; it is associated with high mortality rates [1]. Multidrugresistant $P$. aeruginosa (MDR-PA) and extensively drugresistant $P$. aeruginosa (XDR-PA) strains are becoming major clinical threats worldwide [2]. Recently, it has been estimated that each year in Italy infections due to antibiotic-resistant bacteria exceed 200,000 cases, causing more than 10,000 deaths. Notably, carbapenem- or colistin-resistant Gram-negative species, including $P$. aeruginosa, contributed considerably to the total burden of antibiotic-resistant infections in health care settings [3]. Data from the European Antimicrobial Resistance Surveillance Network (EARS-Net) showed that in 2018, in Italy, the $15.8 \%$ of invasive $P$. aeruginosa isolates were carbapenem-resistant [4]. Moreover, the reported resistance to piperacillin-tazobactam, fluoroquinolones, ceftazidime and aminoglycosides were $23.9 \%, 22.9 \%, 19.9 \%$ and $12.8 \%$, respectively [4].

Infection with sequence type (ST) 235 has been associated with poor clinical outcomes in patients with $P$. aeruginosa bacteremia [5]. In 2013, a unique New Delhi metallo- $\beta$-lactamase-1 (NDM-1)-producing $P$. aeruginosa (NDM-1-PA) ST235 strain was isolated in Italy from a patient with sepsis who had been hospitalized previously in Serbia [6]. In addition, an ST235 NDM-1PA (HIABP11) was isolated in France in 2012 from the urine culture of a patient hospitalized in Serbia 3 months earlier [7]. The present study describes the first Italian case of an autochthonous extensively drug-resistant $P$. aeruginosa strain producing NDM-1- and $\beta$-lactamases isolated from an elderly patient who died from sepsis.

\section{Case presentation}

In August 2019, a 77-year-old woman residing in an elderly health care and rehabilitation facility was hospitalized with chronic respiratory insufficiency caused by chronic obstructive pulmonary disease and pulmonary emphysema in the Internal Medicine ward of San Paolo Hospital in Bari. While hospitalized, she developed a urinary tract infection; $P$. aeruginosa was isolated from a urine sample. No antibiotic was administered. The patient became febrile 3 days later, and $P$. aeruginosa was isolated from blood cultures. She received colistin (a 9 million unit (MU) loading dose, followed by 4.5 MU twice per day) and fosfomycin (6 g every $3 \mathrm{~h}$ ). Following initial improvement, the patient showed a rapid clinical deterioration and died from sepsis in early November.

The biochemical characteristics and antibiotic susceptibility profile of the $P$. aeruginosa isolate were assessed using the automated VITEK 2 system (bioMérieux,
Marcy l'Etoile, France), according to the manufacturer's instructions. The interpretative breakpoints for minimum inhibitory concentrations (MICs) were based on the criteria of the European Committee on Antimicrobial Susceptibility Testing (EUCAST) (http://www. eucast.org/ast_of_bacteria/). The MIC for colistin was determined using the broth microdilution method, as specified by EUCAST guidelines. The phenotypic resistance patterns of $P$. aeruginosa isolated from urine and blood cultures were identical; except for colistin, all isolates were extensively resistant to all tested antimicrobial agents [8]. Strain serotype, corresponding to O11, was determined using monoclonal antibodies and polyclonal antisera (Bio-Rad Laboratories, Milano, Italy). The main carbapenemase-encoding genes $\left(b l a_{\mathrm{KPC}}, b l a_{\mathrm{VIM}}, b l a_{\mathrm{NDM}}\right.$, $b l a_{\mathrm{IMP}}$ and $\left.b l a_{\mathrm{OXA}-48}\right)$ were detected using a commercial multiplex real-time PCR kit (GeneXpert platform, Cepheid, Sunnyvale, CA, USA), which revealed the presence of an NDM gene.

Genomic DNA isolated from a single colony of $P$. aeruginosa was extract using the Qiagen DNeasy Blood and Tissue Kit (Qiagen, Copenaghen, Denmark) and wholegenome sequencing was performed using MiSeq (Illumina San Diego, CA, USA), with a paired-end run of $2 \times 250 \mathrm{bp}$, after Nextera XT paired-end library preparation. De novo genome assembly was performed using SPAdes genome assembler (version 3.12) [9]. The draft genome was approximately $6.941 \mathrm{Mbp}$ in size, with approximately $80 \times$ coverage. The multilocus ST was determined by submitting the assembly to the MLST on-line database (https://pubmlst.org/paeruginosa/). The isolate was assigned to ST235 and named UNIBA_ST235PA (GenBank accession number JAABOY000000000).

The resistome and virulome of the genome were analyzed using ABRicate (https://github.com/tseemann/abricate/). With this tool, a BLAST search of genes included in the Resfinder 3.0 database (https://cge.cbs.dtu.dk/services/ResFinder/) and in the Virulence Factors Database (VFDB) (http://www.mgc.ac.cn/VFs/main.htm) was performed on de novo whole-genome assembly.

ResFinder 3.0 analyses showed that the chromosome of the $P$. aeruginosa isolate harbored several antibiotic resistance genes (Table 1). In addition, the isolate encoded many clinically significant multidrug efflux pump complexes belonging to the resistance nodulation cell division family of $P$. aeruginosa.

Comparative genomic analysis of the NDM-1 regions (12,732 bp) of UNIBA_ST235PA and the P. aeruginosa HIABP11 strain (Acc. No. KC170992) [7] using Geneious R10.2.6 (Biomatters Ltd., USA) showed that these regions were $>98 \%$ identical. The genetic environment surrounding the bla $a_{\text {NDM-1 }}$ gene of UNIBA_ST235PA is displayed in the Fig. 1. An ISCR1 element was located immediately upstream of the chromosomal bla $a_{\mathrm{NDM}-1}$ 
Table 1 Genetic resistance profile of the P. aeruginosa NDM-1-producing strain isolated from a patient hospitalized in Bari, Italy, in October 2019

\begin{tabular}{|c|c|}
\hline Resistance gene product & Resistance genes \\
\hline Aminoglycoside modifying enzymes & 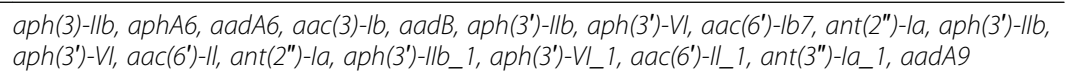 \\
\hline$\beta$-lactamases & bla $a_{\mathrm{NDM}-1}$, bla $a_{\mathrm{PAO}}, b / a_{\mathrm{OXA}-50}, b / a_{\mathrm{OXA}-488}, b l a_{\mathrm{PDC}-2}, b l a_{\mathrm{PDC}-35}$ \\
\hline Fluoroquinolone resistance determinant & $\operatorname{crpP}$ \\
\hline Macrolide resistance determinant & ermE \\
\hline Bicyclomycin resistance determinant & $b c r-1$ \\
\hline Chloramphenicol resistance determinant & catB7 \\
\hline Fosfomycin resistance determinants & fOSA, fOSA4 \\
\hline Triclosan resistance determinants & TriABC-OpmH \\
\hline Multidrug resistance efflux pumps & $\begin{array}{l}\text { MexAB-OprM complex, MexCD-OprJ complex, MexEF-OprN complex, MexGHI-OpmD complex, } \\
\text { MexJK complex, MexMN-OprM complex, MexPQ-OpmE complex, MexWW-OprM complex, } \\
\text { MexXY-OprM complex, MuxABC-OpmB complex, armR, arnA, cpxR, pmpM, gacE-H, gacF, soxR }\end{array}$ \\
\hline
\end{tabular}

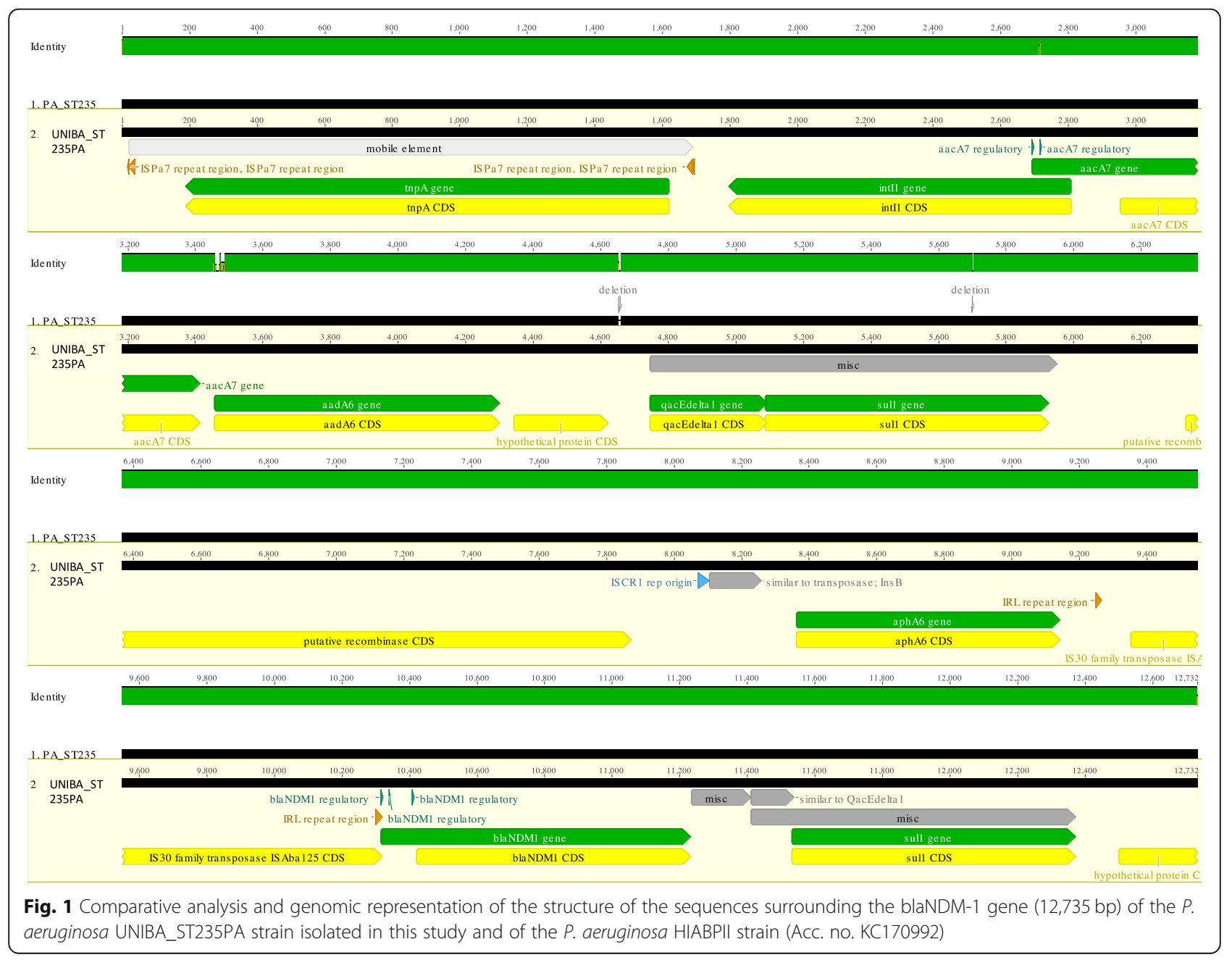


gene, followed by the aminoglycoside resistance gene aphA6; this is identical to that in the HIABP11 strain isolated in France in 2012. In addition, the expression of $b l a_{\mathrm{NDM}-1}$ of UNIBA_ST235PA was under the control of a promoter belonging to the IS30 family transposase ISAba125. Two short deletions were observed in the mapped sequence of UNIBA_ST235PA: an 8 bp deletion in a non-coding region at position 4564 and a 5 bp deletion at position 5699 in the sul1 gene (Fig. 1).

The PlasmidFinder tool (https://cge.cbs.dtu.dk/services/PlasmidFinder/) showed that no plasmids were present in UNIBA_ST235PA.

The virulome of the draft genome identified several virulence determining genes, including the type III secretion system effector ExoU phospholipase, which is associated with a high cytotoxic phenotype, as previously reported $[10,11]$.

\section{Discussion and conclusion}

Preliminary epidemiologic investigation revealed that the patient had no previous history of travel or hospitalization abroad. Further in-depth epidemiological investigation is ongoing to determine the origin and transmission dynamics of this strain; investigations include the screening of all relatives and personnel involved in the care of this patient. Environmental investigations are also underway. All these activities formed part of public health surveillance. Infection control measures, such as an intensive program of environmental cleaning and disinfection, were implemented to reduce the risk of dissemination of this strain and strict contact isolation precautions were applied. Active surveillance was started to monitor emergence of this pathogen also in patients residing in the healthcare and rehabilitation facility.

To the best of our knowledge, this is the first report describing isolation of an autochthonous, extensively drugresistant NDM-1 $P$. aeruginosa ST235 strain in Italy. In Italy, most carbapenem-resistant $P$. aeruginosa strains described to date carry the VIM, IMP, and FIM genes [12]. The only NDM-1-carrying PA was isolated from a stem cell transplant recipient previously hospitalized in Belgrade, Serbia [6]. Of note, the genetic environment surrounding the $b l a_{\mathrm{NDM}-1}$ gene of the present isolate was identical to that of the NDM-1-PAs isolated in France in 2012 and in Italy in $2013[6,7]$.

To date, few strains of NDM-1-PA have been identified worldwide; all that have been identified belong to ST235 [12]. ST235 is a high-risk type associated with MDR/XDR profiles of $P$. aeruginosa strains [2]. The XDR phenotype reduces treatment options significantly, potentially deciding the outcome of $P$. aeruginosa infections [5]. Treatment options are limited for patients infected with XDR strains, which impacts the severity and mortality of these infections [5].
Combination chemotherapy, such as ceftazidimeavibactam and ceftazidime-avibactam-fosfomycin, has shown promise for the treatment of MDR/XDR-PA infections $[13,14]$ but are inactive against metallo- $\beta$ lactamases and some OXA-carbapenemases [15].

Unfortunately, the strain described in the present study shows an XDR pattern, which makes therapeutic options even more limited. Moreover, this strain harbored many virulence genes comprising the ExoU, which is associated with clones of serotype O11-ST235 [16]. The combination of ST235 and ExoU is a predictor of highly unfavorable prognosis [5].

In conclusion, the isolation of this autochthonous $P$. aeruginosa ST235 strain encoding genes producing NDM-1- and $\beta$-lactamases is worrisome. Early identification of high-risk clones could help in investigating outbreaks and controlling infections. WGS of clinical isolates in particular could allow for a better understanding of the spread of resistance markers, and of possible inter-hospital dissemination of specific clones [2]. Continuous monitoring of the development of antibiotic resistance is crucial if we are to better understand local epidemiology and the potential spread of antimicrobial resistance.

\section{Abbreviations}

EUCAST: European Committee on Antimicrobial Susceptibility Testing; MDR: Multidrug-resistant; MICs: Minimum inhibitory concentrations; MU: Million unit; NDM: New Delhi metallo-ß-lactamase; PA: Pseudomonas aeruginosa; ST: Sequence type; VFDB: Virulence Factors Database; XDR: Extensively drug-resistant

\section{Acknowledgements}

Not applicable.

\section{Authors' contributions}

$\mathrm{DL}, \mathrm{MA}$ and $\mathrm{MC}$ conceived and designed the study. AS, VDL performed data collection. ALDR and AM designed and performed the sample testing. LC, LDS and AP analyzed and interpreted the results. MM, MDG and MQ contributed to the design and implementation of control measures. DL, MA and $M C$ wrote and edited the manuscript. MM, MDG and AP incorporated comments, additions and feedback throughout the revision. All authors reviewed the manuscript and approved the final version of the manuscript.

\section{Funding}

Not applicable.

\section{Availability of data and materials}

The nucleotide sequence of UNIBA_ST235PA has been deposited at GenBank under the accession number JAABOY000000000, BioSample SAMN13894995

\section{Ethics approval and consent to participate}

All activities undertaken formed part of public health surveillance (Regional Council Deliberation n. 744 of 15 May 2018) and did not require Ethics approval. An informed written consent was obtained from the legal guardian of the case.

\section{Consent for publication}

Not applicable.

\section{Competing interests}

The authors declared no potential conflicts of interest with respect to the research, authorship, and/or publication of this article. 


\section{Author details}

'Department of Biomedical Sciences and Human Oncology, Hygiene Unit, University of Bari "Aldo Moro", P.zza G. Cesare 11, 70124 Bari, Italy. 2Department of Infectious Diseases, Istituto Superiore di Sanità, Roma, Italy. ${ }^{3}$ Istituto Zooprofilattico Sperimentale della Puglia e della Basilicata, Foggia, Italy. ${ }^{4}$ San Paolo Hospital, ASL, Bari, Italy.

Received: 11 March 2020 Accepted: 9 May 2020

Published online: 25 May 2020

\section{References}

1. Thaden JT, Park LP, Maskarinec SA, Ruffin F, Fowler VG Jr, van Duin D Results from a 13-year prospective cohort study show increased mortality associated with bloodstream infections caused by Pseudomonas aeruginosa compared to other Bacteria. Antimicrob Agents Chemother. 2017;61(6):e02671-16.

2. Oliver A, Mulet X, López-Causapé C, Juan C. The increasing threat of Pseudomonas aeruginosa high-risk clones. Drug Resist Updat. 2015;21-22: 41-59.

3. Cassini A, Högberg LD, Plachouras D, Quattrocchi A, Hoxha A, Simonsen GS, et al. Attributable deaths and disability-adjusted life-years caused by infections with antibiotic-resistant bacteria in the EU and the European economic area in 2015: a population-level modelling analysis. Lancet Infect Dis. 2019;19(1):56-66.

4. European Centre for Disease Prevention and Control. Surveillance of antimicrobial resistance in Europe - Annual report of the European Antimicrobial Resistance Surveillance Network (EARS-Net) 2018. Stockholm: ECDC; 2018. Available from: https://www.ecdc.europa.eu/ sites/default/files/documents/surveillance-antimicrobial-resistanceEurope-2018.pdf.

5. Recio R, Villa J, Viedma E, Orellana MÁ, Lora-Tamayo J, Chaves F. Bacteraemia due to extensively drug-resistant Pseudomonas aeruginosa sequence type 235 high-risk clone: facing the perfect storm. Int J Antimicrob Agents. 2018;52(2):172-9.

6. Carattoli A, Fortini D, Galetti R, Garcia-Fernandez A, Nardi G, Orazi D, et al. Isolation of NDM-1-producing Pseudomonas aeruginosa sequence type ST235 from a stem cell transplant patient in Italy, may 2013. Euro Surveill. 2013;18(46):20633.

7. Janvier F, Jeannot K, Tessé S, Robert-Nicoud M, Delacour H, Rapp C, et al. Molecular characterization of blaNDM-1 in a sequence type 235 Pseudomonas aeruginosa isolate from France. Antimicrob Agents Chemother. 2013;57(7):3408-11.

8. Magiorakos AP, Srinivasan A, Carey RB, Carmeli Y, Falagas ME, Giske $C G$, et al. Multidrug-resistant, extensively drug-resistant and pandrugresistant bacteria: an international expert proposal for interim standard definitions for acquired resistance. Clin Microbiol Infect. 2012;18(3):268-81.

9. Nurk S, Bankevich A, Antipov D, Gurevich AA, Korobeynikov A, Lapidus $A$, et al. Assembling single-cell genomes and minimetagenomes from chimeric MDA products. J Comput Biol. 2013; 20(10):714-37.

10. Hauser AR. The type III secretion system of Pseudomonas aeruginosa: infection by injection. Nat Rev Microbiol. 2009;7(9):654-65.

11. Peña C, Cabot G, Gómez-Zorrilla S, Zamorano L, Ocampo-Sosa A, Murillas J, et al. Influence of virulence genotype and resistance profile in the mortality of Pseudomonas aeruginosa bloodstream infections. Clin Infect Dis. 2015; 60(4):539-48.

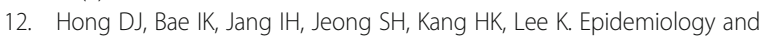
characteristics of Metallo- $\beta$-lactamase-producing Pseudomonas aeruginosa. Infect Chemother. 2015;47(2):81-97.

13. Schaumburg F, Bletz S, Mellmann A, Becker K, Idelevich EA. Comparison of methods to analyse susceptibility of German MDR/XDR Pseudomonas aeruginosa to ceftazidime/avibactam. Int J Antimicrob Agents. 2019;54(2): 255-60.

14. Papp-Wallace KM, Zeiser ET, Becka SA, Park S, Wilson BM, Winkler ML, et al. Ceftazidime-Avibactam in combination with fosfomycin: a novel therapeutic strategy against multidrug-resistant Pseudomonas aeruginosa. J Infect Dis. 2019;220(4):666-76.

15. Nguyen L, Garcia J, Gruenberg K, MacDougall C. Multidrug-resistant Pseudomonas Infections: hard to treat, but Hope on the horizon? Curr Infect Dis Rep. 2018;20(8):23.
16. Recio R, Sánchez-Diener I, Viedma E, Meléndez-Carmona MÁ, Villa J, Orellana MÁ, et al. Pathogenic characteristics of Pseudomonas aeruginosa bacteraemia isolates in a high-endemicity setting for ST175 and ST235 high-risk clones. Eur J Clin Microbiol Infect Dis. 2019. https://doi.org/10.1007/s10096-019-03780-z [Epub ahead of print].

\section{Publisher's Note}

Springer Nature remains neutral with regard to jurisdictional claims in published maps and institutional affiliations.
Ready to submit your research? Choose BMC and benefit from:

- fast, convenient online submission

- thorough peer review by experienced researchers in your field

- rapid publication on acceptance

- support for research data, including large and complex data types

- gold Open Access which fosters wider collaboration and increased citations

- maximum visibility for your research: over $100 \mathrm{M}$ website views per year

At BMC, research is always in progress.

Learn more biomedcentral.com/submissions 